CYBERNETICS AND INFORMATION TECHNOLOGIES • Volume 16, No 5

Special Issue on Application of Advanced Computing and Simulation in Information Systems

Sofia $\bullet 2016$

\title{
Research on QPM and Its Cascaded Structure Applied in FOPA
}

\author{
Xu Ruihong, Chongxiu Yu, Xinzhu Sang, Dahsiung Hsu
}

Institute of Information Photonics and Optical Communications (IPOC), Beijing University of Posts and Telecommunications (BUPT), Beijing, 100876 China

Email: xu_rui_hong@126.com

\begin{abstract}
In the research of optical communication, Optical Parametric Amplification (OPA) has been an important point. As a Four Wave Mixing (FWM) effect based nonlinear process, OPA also requires Phase Matching (PM). Rigorous $P M$ in practical research requires extremely harsh conditions. Quasi Phase Matching (QPM) and its cascaded structure can solve that problem, which would construct an overall phase matching. In the first part of this thesis, a QPM mechanism of segmented High NonLinear Fibre (HNLF) and inserted phase shifter for pumps, was proposed in application of FOPA. The phase shifters would "correct" the phase mismatching after every amplified signal by HNLF section. In this structure the phase matching was always kept in the vicinity of the initial matching value. The signal gain was flatter, and 6.4-9.5 dB higher than that of the non-QPM structure. In second part, a cascaded FWM+OPA structure was used to realize the copier-FOPA according to the cascaded QPM scheme. In the copier part, the information of signal wave was copied into the generated idler wave, before the signal light was amplified. Not only the copy, a $160 \mathrm{~nm}$ (Phase Insensitive, PI)/170 nm (Phase Sensitive, PS) gain bandwidth was obtained, which improved greatly comparing to that of the conventional. In FOPA part, the signal flatter gain decreases by approximately $14 d B(P I) / 15 d B(P S)$, with fluctuation down to $<0.1 d B(P I) /<0.2 d B(P S)$. The gain bandwidth decreases to $135 \mathrm{~nm}(P I) / 150 \mathrm{~nm}(P S)$, which is tens of nanometers wider than conventional FOPA.
\end{abstract}

Keywords: FOPA, QPM, WC, cascaded structure, gain.

\section{Introduction}

Optical Parametric Amplification (OPA) is an important application based on FourWave Mixing (FWM). It is becoming very promising in the research of all optical network system. Compared to other optical amplifications, OPA can reduce the 
influence of phase jitter, reduce the noise to $0 \mathrm{~dB}$ theoretically, suppress the modulation instability, and regenerate the phase of signal wave, et al. OPA can be realized both in fibre and other waveguide like Semiconductor On Insulator (SOI). In this thesis, the OPA research of bandwidth and noise are all based on fibre.

Conventional highly efficient FWM effect needs the Phase Matching (PM), accurately. However, practical result shows the theoretical requirement is strictly limited. Quasi Phase Matching (QPM) technique comes to the research point in decades, in order to overcome that limitation [1, 2]. First introduced by Armstrong Blembergen at al. [3] in 1962, QPM technology refers to the adjustment of the relative phase among the light waves in the process of transmission, either periodically or non-periodically. It is equivalent to a mechanism of "correction". Even phase mismatches badly, QPM can compensate the deterioration by adjusting the phases. Therefore, comparing to the conventional PM technology, QPM is more feasible and flexible. Moreover, various QPM solutions can be designed by different parameter demand.

In previous studies, QPM is the periodic modulation of crystal nonlinear susceptibility to compensate the phase mismatch caused by refractive index dispersion. In other word, QPM realize in periodically poled crystal. In following studies, QPM is not limited to the periodic propagation properties of crystals any more. By constructing suitable light path and device, QPM can be achieved in form of sectional phase matching.

In the first part of this thesis, a QPM structure will be designed in FOPA. In the second part, a cascaded structure of Wavelength Conversion (WC) and FOPA which similar to QPM is designed to realise a better gain performance than conventional FOPA.

\section{Phase matching in OPA}

Take a typical one signal (input), one pump (input), two idler wave (sideband, output) model as example. The analytic expression of propagation field in fibre can be variable separated as

$$
\begin{gathered}
E(x, y, z)=f(x, y) A(z)=\frac{1}{2} f(x, y)\left[A_{\mathrm{p}}(z) \exp j\left(\beta_{\mathrm{p}} z-\omega_{\mathrm{p}} t\right)+\right. \\
\left.+A_{\mathrm{s}}(z) \exp j\left(\beta_{\mathrm{s}} z-\omega_{\mathrm{s}} t\right)+A_{i}(z) \exp j\left(\beta_{i} z-\omega_{i} t\right)+c . c\right] .
\end{gathered}
$$

For not confused with the idle wave $i, j$ represents the imaginary unit; $f(x, y)$ is the transverse distribution; c.c represents complex conjugation which is negligible in calculation. With the attenuation part neglected, respective amplitude coupling equations can be derived as:

$$
\begin{aligned}
& \text { (2) } d A_{1} / d z=j \gamma\left[\left|A_{1}\right|^{2} A_{1}+2\left(\left|A_{2}\right|^{2}+\left|A_{3}\right|^{2}+\left|A_{4}\right|^{2}\right) A_{1}+2 A_{3} A_{4} A_{2}^{*} \exp (j \Delta \beta z)\right], \\
& \text { (3) } d A_{2} / d z=j \gamma\left[\left|A_{2}\right|^{2} A_{2}+2\left(\left|A_{1}\right|^{2}+\left|A_{3}\right|^{2}+\left|A_{4}\right|^{2}\right) A_{2}+2 A_{3} A_{4} A_{1}^{*} \exp (j \Delta \beta z)\right],
\end{aligned}
$$


(4) $d A_{3} / d z=j \gamma\left[\left|A_{3}\right|^{2} A_{3}+2\left(\left|A_{1}\right|^{2}+\left|A_{2}\right|^{2}+\left|A_{4}\right|^{2}\right) A_{3}+2 A_{1} A_{2} A_{4}^{*} \exp (j \Delta \beta z)\right]$,

(5) $d A_{4} / d z=j \gamma\left[\left|A_{4}\right|^{2} A_{4}+2\left(\left|A_{1}\right|^{2}+\left|A_{2}\right|^{2}+\left|A_{3}\right|^{2}\right) A_{4}+2 A_{1} A_{2} A_{3}^{*} \exp (j \Delta \beta z)\right]$,

where the nonlinear coefficient $\gamma=2 \pi n_{2} / \lambda A_{\text {eff }}=n_{2} \omega / c A_{\text {eff }}, n_{2}$ is the effective refractive index, $A_{\text {eff }}$ is the effective mode-field area. The first item on the right side of the equations represents self-phase modulation process, while the second represents cross phase modulation process. The third item is the parametric process, which realizes the optical parametric amplification. At a power standpoint, it expresses the energy transfer among the waves.

We can solve the analytical solution of input waves in (2) and (3):

$$
\begin{aligned}
& A_{1}(z)=\sqrt{P_{1}} \exp \left[j \gamma z\left(P_{1}+2 P_{2}\right)\right], \\
& A_{2}(z)=\sqrt{P_{2}} \exp \left[j \gamma z\left(P_{2}+2 P_{1}\right)\right] .
\end{aligned}
$$

Let $n=1,2,3,4$. Then (2)-(5) can be rewritten as [4]:

$$
\begin{gathered}
d P_{1,2} / d z=-2 \gamma \sqrt{P_{1} P_{2} P_{3} P_{4}} \sin \theta, \\
d P_{3,4} / d z=2 \gamma \sqrt{P_{1} P_{2} P_{3} P_{4}} \sin \theta,
\end{gathered}
$$

where the relative phase difference is

$$
\theta(z, t)=\Delta \beta z+\phi_{1}(z)+\phi_{2}(z)-\phi_{3}(z)-\phi_{4}(z)+\omega_{1} t+\omega_{2} t-\omega_{3} t-\omega_{4} t .
$$

Partial derivation of (10) will be

$$
\begin{gathered}
\partial \theta / \partial z=\Delta \beta+\gamma\left(P_{1}+P_{2}-P_{3}-P_{4}\right)+ \\
+\gamma\left[\sqrt{P_{1} P_{2} P_{3} / P_{4}}+\sqrt{P_{1} P_{2} P_{4} / P_{3}}-4 \sqrt{P_{3} P_{4}}\right] \cos \theta,
\end{gathered}
$$

where $\Delta \beta$ represents the linear phase shift, while

$$
\gamma\left(P_{1}+P_{2}-P_{3}-P_{4}\right) \text { and } \gamma\left[\sqrt{P_{1} P_{2} P_{3} / P_{4}}+\sqrt{P_{1} P_{2} P_{4} / P_{3}}-4 \sqrt{P_{3} P_{4}}\right] \cos \theta,
$$

represent the nonlinear phase shift.

According to (8) (9) and (11), by changing the relative phase difference:

when $0<\theta \leq \pi / 2, d P_{1,2} / d z<0$ and $d P_{3,4} / d z>0$; power flows from pumps to signal and idler waves; that is the process of OPA;

when $-\pi / 2 \leq \theta<0, d P_{1,2} / d z>0$ and $d P_{3,4} / d z<0$; power flows in a counter direction, where the power of signal attenuates.

Thus, to fulfill the amplification of signal, $\theta$ should stay around $\pi / 2$, which means $\cos \theta \approx 0$. Equation (11) can be simplified in condition of intense pump $\left(P_{1,2} \gg P_{3,4}\right)$ :

$$
\partial \theta / \partial z=\Delta \beta+\gamma\left(P_{1}+P_{2}-P_{3}-P_{4}\right) \approx \Delta \beta+\gamma\left(P_{1}+P_{2}\right)=\Delta \beta+\gamma P_{p}=\kappa,
$$

where $\kappa$ is the total phase mismatch (phase matches totally when $\kappa=0$ ). 


\section{A cascaded structure of QPM technique with HNLF and phase shifter for FOPA}

\subsection{Structure}

Narrow gain bandwidth and peak-shape gain spectrum [5] are always the limitations in the current FOPA research. One factor limiting flat broadband gain mainly refers to phase mismatch, caused by dispersion instability along the propagation direction, is more likely to occur in fibre than in semiconductor (such as Semiconductor On Insulator, SOI). QPM is more urgent to achieve in fibre [6]

The conventional QPM technique research mostly focused on the periodic phase mismatch compensation mechanism caused by the change of the crystal structure. Being different from periodic nonlinear optical crystal with second order nonlinear susceptibility $\chi^{(2)}$, there is only third-order nonlinear susceptibility $\chi^{(3)}$ in fibre. However, QPM can be realized in fibre by changing the phases. Here the degenerated form of (10) can explain the scheme

$$
\Phi(z)=\phi_{\mathrm{s}}+\phi_{i}-2 \phi_{\mathrm{p}}+\left(\beta_{\lambda_{\mathrm{s}}}+\beta_{\lambda_{i}}-2 \beta_{\lambda_{\mathrm{p}}}\right) z .
$$

A cascaded structure can manage $\Phi(z)$, shown as in Fig. 1. High NonLinear Fibre (HNLF) or Photonic Crystal Fibre (PCF) is a proper selection for FWM process. The theory of this structure is that a pump phase shifter forces $\Phi(z)$ returning zero after a section of HNLF or PCF. The phase shifter shifts only the phase corresponding to the wavelength of the pump wave. In the whole process, $\Phi(z)$ is managed to keep below a threshold $(<\pi)$, so that the phase mismatch is small enough to achieve high gain.

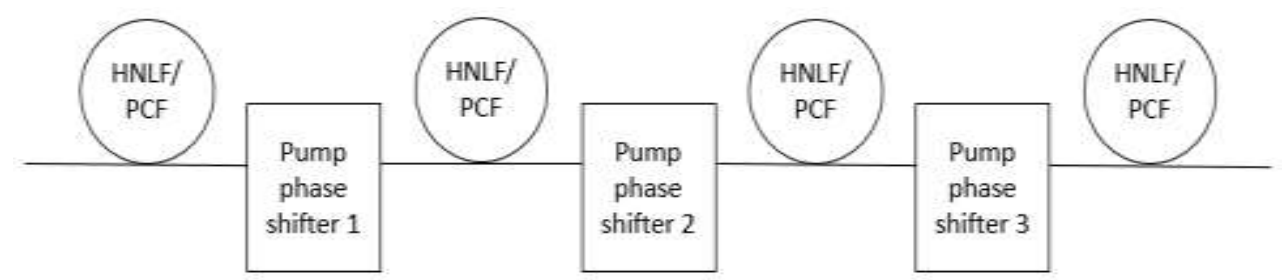

Fig. 1. The cascaded QPM to realize FOPA

\subsection{Results and analysis}

The parameters used in the simulation are as followed.

The pump wavelength and the zero dispersion wavelength of fibre $\lambda_{\mathrm{p}}=\lambda_{0}=1565 \mathrm{~nm}$. The signal wavelengths $\lambda_{\mathrm{s} 1}=1540 \mathrm{~nm}, \lambda_{\mathrm{s} 2}=1550 \mathrm{~nm}$ and $\lambda_{\mathrm{s} 3}=1560 \mathrm{~nm}$, for comparing the performance. The pump power $P_{\mathrm{p}}=32 \mathrm{~dB} . \mathrm{m}$. The signal power $P_{\mathrm{s}}=-20 \mathrm{~dB} . \mathrm{m}$. The length of very HNLF (or PCF) section $L_{i}=50 \mathrm{~km}$, $i=1,2,3,4$ (which means three pump shifters).

The relative phase varying along the transmission comparing to non-QPM situation is shown in Fig. 2. 


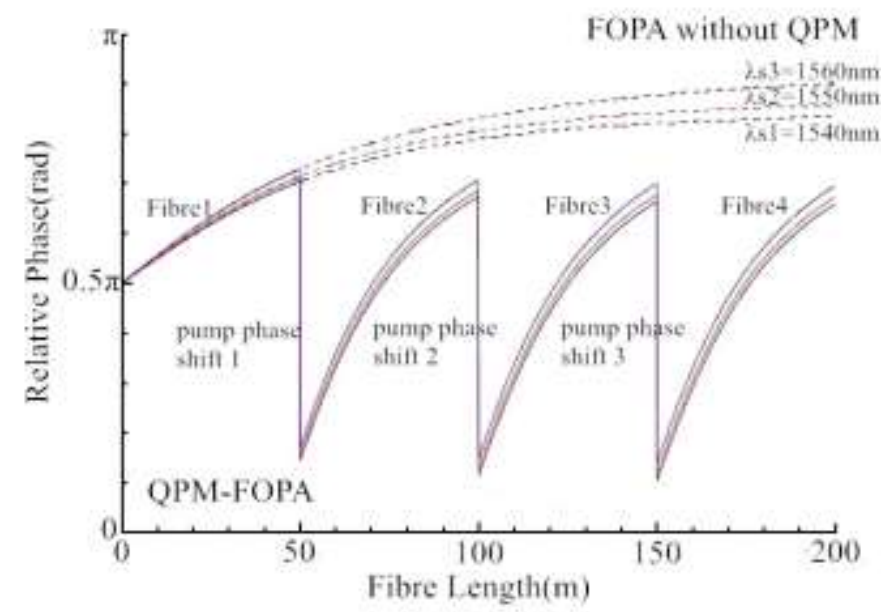

Fig. 2. The comparison of relative phase between conventional FOPA and QPM-FOPA

According to (12) and (13), the initial relative phase $\Phi(0)$ was set to be $0.5 \pi$. The pump phase shifter "correct" the phase relation, timely. By comparing the phase characteristic of different signal wavelengths of $1540 \mathrm{~nm}, 1550 \mathrm{~nm}$ and $1560 \mathrm{~nm}$, both in QPM and conventional FOPA, Fig. 2 shows the following results:

(1) In conventional FOPA, the relative phase shifts upward to $\pi$ ( $0.9 \pi$ or so), while severe phase mismatch will cause the deterioration of signal amplification. With the increase of the transmission distance along the optical fibre, the phase shift between different wavelengths will show obvious difference. That is a limitation of the amplification bandwidth.

(2) Because of the pump shift when relative phase reaches a certain value, the phase is "forced" to carry out a matching, to keep it around the initial $0.5 \pi$, which is a necessary condition of FOPA phase matching. The signal gain varying with transmission also confirms the two points above, as shown in Fig. 3.

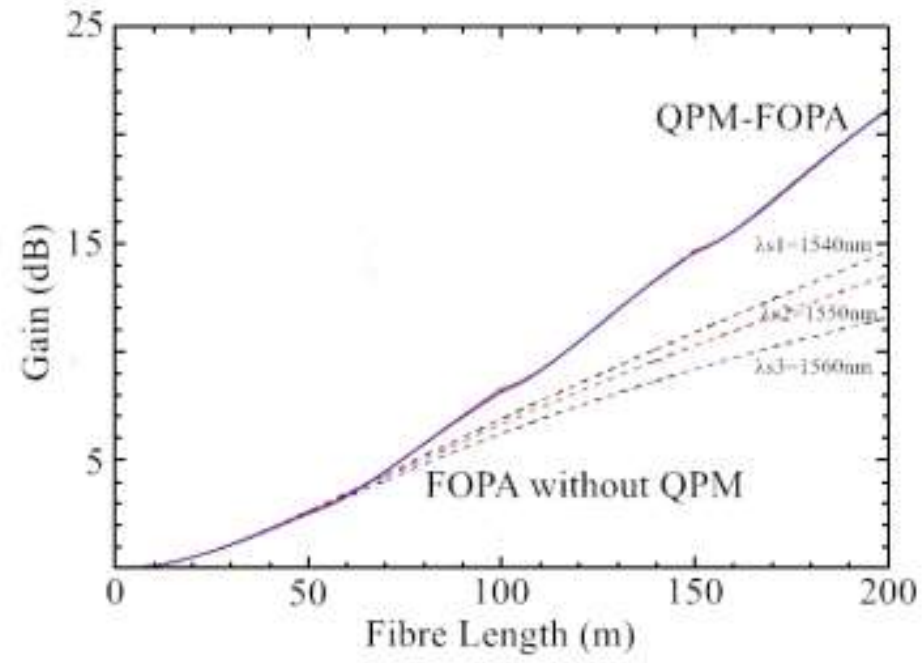

Fig. 3. The comparison of signal gain between conventional FOPA and QPM-FOPA 
Fig. 3 shows the following results:

(1) As the transmission characteristic differs from the corresponding wave lengths along the fibre, the relative phase among signal, pump and idler waves will increase rapidly. Then the phase mismatching will be quite different from each other. The signal gains appear different slope. With the "correction" of QPM shift the pump phase uniformly, signal waves with different wavelengths keep the same growth, which present a flat gain in output.

(2) By the sectional compensation of phase matching, QPM-FOPA provides a higher gain than convention. Shown in Fig. 3, in a $200 \mathrm{~m}$ transmission length, the gain of QPM-FOPA is 6.4-9.5 dB higher than that of conventional FOPA.

\section{A cascaded structure of WC and OPA for FOPA}

\subsection{Structure}

The research in recent years classified OPA into Phase Sensitive (PS-OPA) and Phase Insensitive (PI-OPA). PI-OPA amplifies all the waves on demand. PS-OPA[7-9] is the process in which waves with the same phase to signal are amplified, while in which waves with the phase different from signal are not amplified; or even been eliminated. If the phase noise of signal wave is eliminated in amplification, PI-OPA will play an important part in the performance of improving deteriorated signal.

Generating the idler wave before parametric amplification is an effective technique in PS-OPA. Fig. 4 shows a theoretical PS-OPA structure with generating idler ahead of amplification, which is called "copier" structure [10-12]. The copier process is actually a process of wavelength conversion. It realizes a function of copying the information of signal wave to idler wave. The information is transmitted in two wavelengths, and finally overlapped in receiver. The advantages of this "pre-conversion" are as follows:

(1) The signal, pump and idler waves are automatically phase-locked. Any modulated signal wave can be PS-amplified, without considering the relative phase of the input waves.

(2) The signal waves with its corresponding idler waves can share a common pump wave.

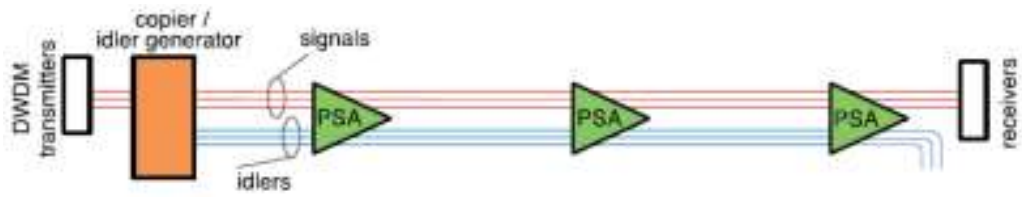

Fig. 4. The theoretical structure of copier-PS-OPA(WC+OPA) [12]

Advanced to the single wavelength transmission for information, this structure will provide a $3 \mathrm{~dB}$ improvement to signal wave at the receiver side [11]. If the phase sensitivity needs to be improved in PS-OPA, there should be an attenuation between the WC and OPA processes. The attenuation decreases the correlated noise generated by WC part. However, the noise de-correlation is realized usually by the 
attenuation of the link. This attenuation decreases the Noise Figure (NF) of the whole link; but the transmission attenuation is inevitable. Therefore, once the fibre is longer than $50 \mathrm{~km}$, without any extra attenuation, the phase sensitivity can keep increasing.

Combining the cascaded amplification and PS-OPA, am amplification with two PM-HNLF (Polarization Maintaining-) is considered. The first HNLF realize the wavelength conversion, for copy the information of signal to generated conjugated idler wave. The second HNLF amplifies the signal wave. Fig. 5 shows the theoretical structure of it.

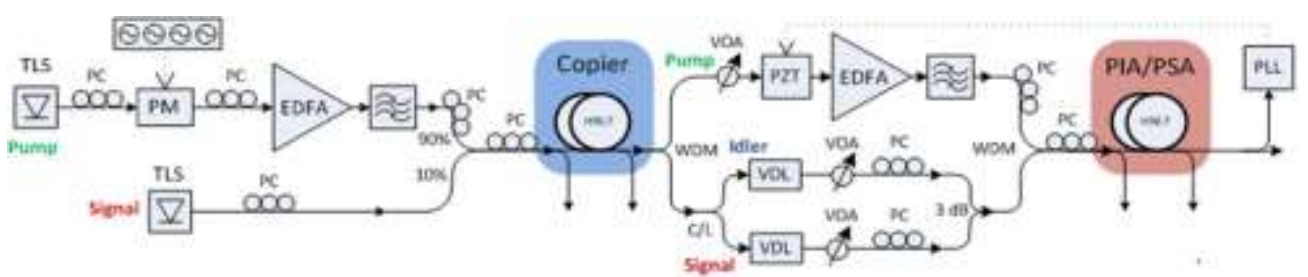

Fig. 5. The cascaded structure of WC and OPA. TLS is the tunable laser source; PM is the phase modulator; VDL is the variable delay line; VOA is the variable optical attenuation;

PLL is the phase-locking loop

The first WC part realizes the copy of conjugated idler wave. The pump wave can be generated by TLS, then phase-modulated, pre-amplified by EDFA. Variable BPF with $1 \mathrm{~nm}$ bandwidth would filter most of the Amplified Spontaneous Emission Noise (ASEN) around the wavelength of pump. The signal wave is generated by another TLS, with wavelength ranging from 1580 up to $1680 \mathrm{~nm}$. After a 90/10 coupler, waves are in the first HNLF. Here the signal is copied to the idler wave. The output waves of the first HNLF are signal and conjugated idler.

The second realize the function of OPA. WDM separates the pump wave from signal and idler waves. The $\mathrm{C}+\mathrm{L}$ waveband $(1530-1565 \mathrm{~nm}+1565-1625 \mathrm{~nm})$ coupler separates the signal and idler waves. The attenuation discriminating the noise, pump wave is amplified again by EDFA. The PieZoelectric-Transducer (PZT) provide the phase shift [5]. This process needs a feedback from PLL, which locked the relative phase of pump and signal/idler wave, and avoiding disturbing by temperature and mechanical characteristic. For idler wave, VDL controls its temporal calibration and VOA controls its power for fear of low OSNR. The VDL and VOA keep the idler and signal waves in the same format. Finally by WDM and $3 \mathrm{~dB}$ coupler, all the waves are coupled into the second HNLF for PSA. The output of the PSA needs to be a feedback for eliminating the pump power.

\subsection{Results and analysis}

The length of the first HNLF is $90 \mathrm{~m}$. Pump wavelength $\lambda_{\mathrm{p}}=1563.3 \mathrm{~nm}$, pump power $P_{\mathrm{p}}=20 \mathrm{~dB} . \mathrm{m}$. Signal power $P_{\mathrm{s}}=-25 \mathrm{~dB} . \mathrm{m}$. The zero dispersion wavelength of HNLF $\lambda_{0}=1562.6 \mathrm{~nm}$. Higher order dispersion $\beta_{3}=4.5 \times 10^{-41} \mathrm{~s} / \mathrm{m}$, $\beta_{4}=9.5 \times 10^{-56} \mathrm{~s} / \mathrm{m}$. The nonlinear coefficient is $10 \mathrm{~W}^{-1} \cdot \mathrm{km}^{-1}$. The simulation research was carried out in both PI- and PS-situation. Fig. 6 shows the signal gain characteristic by copying the conjugated idler wave in the first HNLF. The 
maximum of PIA gain is $20 \mathrm{~dB}$, while PSA is $25 \mathrm{~dB}$. If $10 \mathrm{~dB}$ is set as the gain cutoff, the corresponding gain bandwidths are $160 \mathrm{~nm}$ and $170 \mathrm{~nm}$. The FWHM bandwidth is around $110 \mathrm{~nm}$.

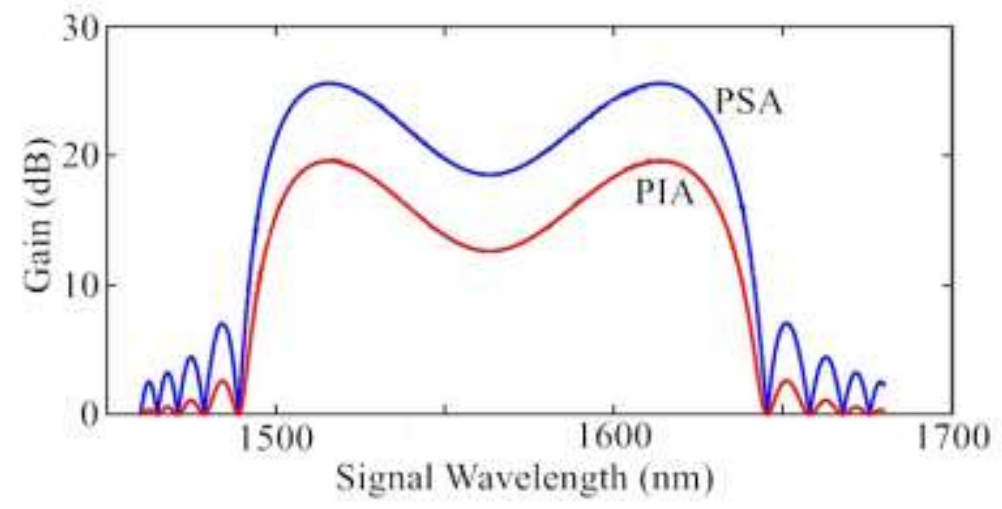

Fig. 6. Gain varies with signal wavelength in the first HNLF (WC, idler copier)

The length of the second HNLF is $45 \mathrm{~m}$. As expected, in shorter HNLF the gain curve is flatter (gain fluctuation $<0.2 \mathrm{~dB}$ of PSA, gain fluctuation $<0.1 \mathrm{~dB}$ of PIA). The gain difference among is much less than that in the first HNLF. The peak gain of PIA and PSA decrease $14 \mathrm{~dB}$ and $15 \mathrm{~dB}$, respectively. If $0 \mathrm{~dB}$ is set to be the gain cut off, the gain bandwidth is $135 \mathrm{~nm}$ and $150 \mathrm{~nm}$, respectively. FWHM bandwidth increases to $120 \mathrm{~nm}$ on the contrary. Fig. 7 shows the result.

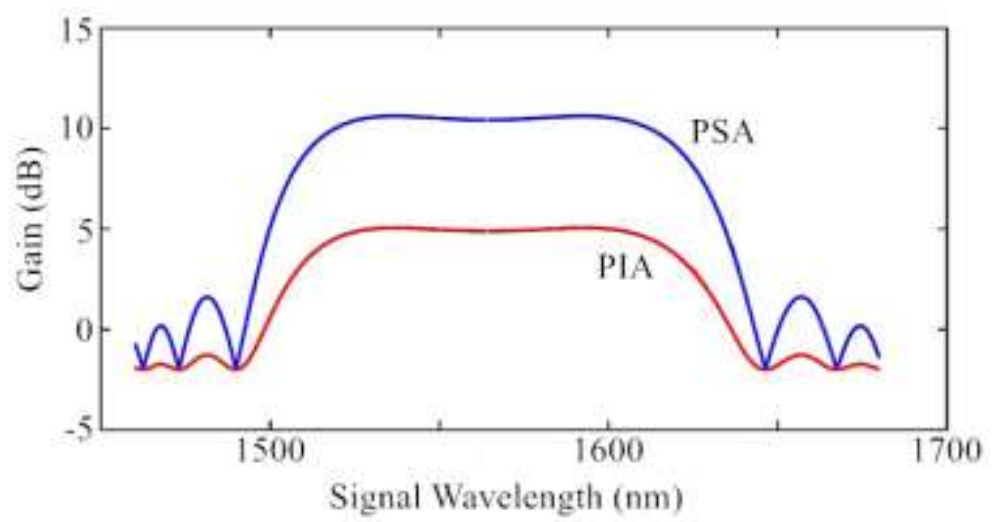

Fig. 7. Gain varies with signal wavelength in the second HNLF (OPA)

\section{Conclusion}

In this thesis, on demand of PM in FOPA, the QPM technique was applied in FOPA. By inserting a pump phase shifter in sections, signals amplified in corresponding HNLF or PCF would be "compensated" in phase matching. That leads to a higher gain, with flatter gain curve.

With the similar design, a cascaded structure of WC and OPA was proposed and simulated. The WC part generated idler, copied the information into idler and 
pre-amplified the signal. The signal was amplified with a similar gain curve, which is not that flat at the gain peak. The OPA part amplified the signal again, to obtain a flatter gain peak.

Acknowledgment: This work is supported by National High-tech R\&D Program (863 Program, Grant No 2013AA031501).

\section{References}

1. Lefevre, Y., N. Vermeulen, H. Thienpont. Quasi-Phase-Matching of Four-WaveMixing-Based Wavelength Conversion by Phase-Mismatch Switching. - J. Lightw. Technol. Vol. 31, July 2013, No 13, pp. 2113-2121.

2. Verme u l e n, N., J. E. S i p e, H. Th i e n p o n t. Quasi-Phase-Matched Cavityenhanced Raman Converter Based on a Silicon Nanowire Ring. - IEEE Photon. Technol. Lett., Vol. 22, December 2010, No 24, pp. 1796-1798.

3. B lo e mberge n, A. N., J. D c u ing et al. Interaction between Light Waves in a Nonlinear Dielectric. - Physics Review, Vol. 127, 1962, No 6, pp. 1918-1939.

4. V altarescu. Light Conversion in Nonlinear Single-Mode Optical Fibers. - J. Lightwave Technol., Vol. 5, 1987, pp. 1652-1659.

5. In o u e, K., T. Muk k i. Signal Wavelength Dependence of Gain Saturation in a Fiber Optical Parametric Amplifier. - Opt. Lett., Vol. 26, 2001, pp. 10-12.

6. Han sryd, J., P. A. Andre k s on, M. We st l und, J. Li, P.-O. He d e kvi st. Fiber-Based Optical Parametric Amplifiers and Their Applications. - Journal Selected Topics Quantum Electronics, Vol. 8, 2002, Issue 3, pp. 506-520.

7. Takas ak a, S., Y. Mi mura, M. Takah a shi, R. Sugizaki, H. Og o shi. Flat and Broad Amplification by Quasi-Phase-Matched Fiber Optical Parametric Amplifier. - In: Proc. of Optical Fiber Communication Conference, 2012, OTh1C.4.

8. Karlsso n, M. Four-Wave Mixing in Fibers with Randomly Varying Zero Dispersion Wavelength. - J. Opt. Soc. Amer. B, Vol. 15, 1998, pp. 2269-2275.

9. Levenson, J. A., I. A bram, T. Rivera, P. Grangier. Reduction of Quantum Noise in Optical Parametric Amplification. - J. Opt. Soc. Amer. B, Vol. 10, 1993, pp. 2233-2238.

10. I maju k u, W., A. Takada. Gain Characteristics of Coherent Optical Amplifiers Using a Mach-Zehnder Interferometer with Kerr Media. - IEEE J. Quantum Electron., Vol. 35, November 1999, pp. 1657-1665.

11. T a n g, R., P. D e vg a n, P. L. V o s s, V. S. Grig or y a n, P. Ku m a r. In-Line FrequencyNondegenerate Phase-Sensitive Fiber-Optical Parametric Amplifier. - IEEE Photon. Technol. Lett., Vol. 17, September 2005, No 9, pp. 1845-1847.

12. Tong, Z., C. Lund ström, E. Tip suw annakul, M. Karls son, P. A. Andrekson. Phase-Sensitive Amplified DWDM DQPSK Signals Using Free-Running Lasers with 6-dB Link SNR Improvement over EDFA-Based Systems. - In: Proc. of European Conf. Exhib. Optical Communication, Torino, Italy, September 2010, Paper PDP1.3.

13. Tong, Z., C. Lundströ m, P. A. Andrekson, C. J. Mc Kinstrie, M. Karls son, D. J. B lessing, E. Tipsuwannakul, B. J. Puttnam, H. Toda, L. Gruner$\mathrm{N}$ i e ls e n. Towards Ultrasensitive Optical Links Enabled by Low-Noise Phase-Sensitive Amplifiers. - Nature Photon., Vol. 5, Juny 2011, No 7, pp. 430-436.

14. Corcoran, S. L. I. O1s s on, C. Lundströ m, M. Karls s on, P. Andrekson. PhaseSensitive Optical Pre-Amplifier Implemented in an $80 \mathrm{~km}$ DQPSK Link. - In: Proc. of Opt. Fiber Commun. Conf. Expo. Nat. Fiber Optic Eng. Conf. (Optical Society of America, Washington, DC, 2012), PDP5A.4. 